\title{
Zur Rolle der Medienpädagogik im Inklusionsprozess
}

\author{
Ingo Bosse \\ Technische Universität Dortmund
}

Ebenso wie das Thema Inklusion ist das Lernen mit Medien ein pädagogischer Megatrend der letzten Jahre. Beide Entwicklungen bringen große Veränderungen in Bildungseinrichtungen und rufen damit gleichermaßen große Erwartungen wie Befürchtungen hervor. Die Frage, welchen Beitrag die Medienpädagogik zur Partizipation sozial Benachteiligter leisten kann, ist dabei nicht neu. Zentrale Figuren der deutschsprachigen Medienpädagogik wie Horst Niesyto haben sich von jeher damit befasst (Maurer u.a. 2013). Diese Perspektive wurde in den letzten Jahren durch den expliziten Einbezug der Gruppe von Menschen mit Behinderungen aber deutlich erweitert, und neben dem Begriff der Partizipation spielt nun ebenso der Inklusionsbegriff eine zentrale Rolle. „Full and effective participation and inclusion in society" (UN 2006, 5) ist das erklärte Ziel der 2006 veröffentlichten UN-Behindertenrechtskonvention. Sie beschreibt den Einsatz von Medien als wesentlich für die Umsetzung der Grund- und Menschenrechte von Menschen mit Behinderung. Diesen Impuls aufgreifend gehört die Medienpädagogik heute $\mathrm{zu}$ den erziehungswissenschaftlichen Teildisziplinen, die sich sehr intensiv mit dem Inklusionsprozess auseinandersetzen (Maurer u. a. 2013). Dabei steht sowohl die außerschulische als auch die schulische Medienbildung im Fokus. Die Bedeutung der Medienbildung für den Inklusionsprozess wird zum Beispiel in den Leitlinien der Deutschen UNESCO Kommission zur Ausgestaltung eines inklusiven Schulsystems deutlich: „Es ist ausgestattet und in der Lage, Vielfalt zu begegnen durch: ... Flexible Lehrmethoden mit innovativen Ansätzen für Unterrichtsmittel, Ausstattung und den Einsatz von Informations- und Kommunikationstechnologien." (Malina 2009, 15) Die Potenziale und Chancen, den Inklusionsprozess mithilfe der Medienpädagogik aktiv mitzugestalten, sind aber bislang nur unzureichend geprüft. Erfahrungen aus Ländern, die eine längere Tradition der inklusiven Beschulung haben und zugleich als innovativ in Medienbildung gelten, zeigen, wie Medien den Inklusionsprozess auf vielfältige Weise unterstützen können (Florian/Hegarty 2004; Richiger-Näf 2010).

Zentral für die Diskussion sind die Begriffe Medienkompetenz und Medienbildung. Hier soll der Argumentation von Schorb und Wagner $(2013,19)$ gefolgt werden: „Medienkompetenz bestimmt sich inhaltlich aus der Perspektive, Menschen als aktiv Gestaltende ihrer Lebensführung und als aktiv Handelnde im Umgang mit Medien anzusehen. Da die Möglichkeiten zunehmen, sich mit und über Medien auszudrücken, zu kommunizieren und zu interagieren, steigen auch die Anforderungen an die Fähigkeiten der Subjekte, mit diesen Medien umzugehen. Dies betrifft die Kinder und Jugendlichen selbst, aber auch ihr familiäres und soziales Umfeld sowie ihre pädagogischen Bezugspersonen. Da die Medien zunehmend den Alltag durchdringen, ist ein kompetenter Umgang mit Medien notwendig, um ein souveränes und selbstbestimmtes Leben füh- 
ren zu können. " Zur grundlegenden Differenzierung der Begriffe Medienkompetenz und Medienbildung wird dem Verständnis Tulodzieckis gefolgt. Er beschreibt Medienkompetenz als Ziel und Medienbildung als Prozess, der den Rahmen für die Förderung von Medienkompetenz vorgibt. Der Terminus Medienbildung findet demnach Verwendung, wenn es um eine inhaltliche Strukturierung der Medienkompetenz geht (Tulodziecki 2010, 45). Inklusive Medienbildung setzt Medienbildung für inklusive Gruppen um. Dabei geht es um die Verwirklichung des Rechts auf uneingeschränkte Teilhabe an der Informationsgesellschaft, der mediatisierten Welten und der Kommunikationskultur unabhängig von persönlichen Prädispositionen wie z. B. einer Beeinträchtigung, ergo um Medienbildung für alle! (Bosse 2013) Die Förderung von Medienkompetenz wird als Beitrag zur Inklusionsbewegung betrachtet, als weitere Möglichkeit des gemeinsamen Lernens bei heterogenen Lernausgangslagen (Michaelis/Lieb 2006). Durch die Stärkung der Medienbildung in allen Bereichen, in denen "das informelle und wechselseitige Lernen behinderter und nicht behinderter Menschen voneinander stattfindet" (Körner 2006, 11), kann Inklusion ermöglicht bzw. erleichtert werden. Es geht darum, die Medienkompetenz aller Heranwachsenden zu stärken und dabei wechselseitige Lernprozesse zu befördern.

Die grundlegende Prämisse einer Medienbildung, die auch die besonderen Bedürfnisse behinderter Schüler/innen berücksichtigt, lautet, „Technologien aus der alleinigen, funktionalen Verknüpfung mit Hilfsmitteln zu lösen und für sinnvolle pädagogische Anwendungskontexte zu öffnen“" (Schnaak/Böhmig 2012, 21). Sie eröffnet allen ein möglichst großes Maß an Selbstständigkeit. Angebote sollen so gestaltet werden, dass Medienbildung für alle eine Bereicherung darstellt. Menschen mit Behinderungen haben sehr unterschiedliche Voraussetzungen und Bedürfnisse, dementsprechend vielfältig sind auch die Konzepte inklusiver Medienbildung. Grundsätzlich ist inklusive Medienbildung nutzerfreundlich. Das bedeutet, es müssen Methoden und Erklärungsweisen gefunden werden, die allgemein verständlich sind. Der jeweilige Kompetenzrahmen muss dann individuell adaptiert werden. Prinzipiell werden eine leicht verständliche Sprache, allgemeingültige Pictogramme und eindeutiges Bildmaterial verwendet. Inklusive Medienbildung liefert Erklärungen und eindeutige Handlungsanweisungen und berücksichtigt neben der Sprachebene auch das Lernen auf auditiver, haptischer und visueller Ebene. Formen aktiver Medienarbeit, die handlungs- und produktionsorientiert vorgehen, sind zu bevorzugen. Voraussetzung für die inklusive Medienbildung ist zunächst der Zugang zu Medien ohne Hindernisse - ergo Barrierefreiheit. „Die Behindertenrechtskonvention (Artikel 20 und 23) wie auch die Inklusionspädagogik, etwa der ,Index für Inklusion' (Booth/Ainscow 2003), fordern gleichermaßen Barrierefreiheit. Sie richten den Blick nicht auf die Defizite des Individuums, sondern auf die Defizite der Umwelt. Ein inklusiver Unterricht ist gehalten, alle Barrieren für Lernen und Teilhabe zu beseitigen. (...) Im inklusiven Kontext kommt es vor allem darauf an, angemessene Vorkehrungen für eine barrierefreie Information und Kommunikation zu treffen." (Wocken 2011, 127) Kernanliegen ist die informationelle Partizipation. Dazu kann die Medienpädagogik einen entscheidenden Beitrag leisten. Informationelle Partizipation bedeutet weit mehr als barrierefreies Webdesign, wenngleich dies inzwischen eine große Rolle spielt $^{1}$. Das Gesamtziel informationeller Partizipation besteht darin, alle Informations- und Kommunikationssysteme so zu gestalten, dass sie für alle Menschen unabhängig von Hardware, Software, Sprache, Kultur, Ort, physischen oder kognitiven Fähigkeiten nutzbar sind (Caspers 2011). Wesentliche Fortschritte konnten in den letzten Jahren durch Innovationen im Bereich unterstützender Technologien erzielt werden². 
Weiter folgt inklusive Medienbildung den Maximen „universellen Designs“. Dieses wird in der UN-Behindertenrechtskonvention von „Produkten, Umfeldern, Programmen und Dienstleistungen“ (UN 2008) gefordert. Das heißt, sie sollen von allen Menschen möglichst weitgehend ohne eine spezielle Anpassung nutzbar sein. Im Kontext der Nutzung moderner Informations- und Kommunikationsmedien ist das Ziel eines universellen Designs die Sicherstellung, dass Informationstechnologien und Telekommunikation für alle Bürger verfügbar und nutzbar sind. Eine nachträgliche Anpassung oder ein spezielles Design sollen verhindert werden (Stephanidis 2001). Der Abbau von Barrieren ist nicht ausschließlich für Menschen mit Behinderung bedeutsam, sondern käme allen Bürgern zugute (Ottinger 2008).

Barrierefreiheit bezieht sich nicht nur auf die Zugänglichkeit und Nutzbarkeit von Lernmaterialien in technischer Hinsicht, sondern auch auf sprachliche Barrierefreiheit. In Bildungsinstitutionen ist nach wie vor eine mittelschichtsorientierte Sprache maßgebend. Diese stellt für Schüler mit Lernschwierigkeiten häufig ein Lernhindernis dar, ebenso wie der monolinguale Habitus für Heranwachsende mit Zuwanderungsgeschichte. Beide Gruppen benötigen einfache Sprache. „Alle Lernmaterialien und -medien (Schulbücher, Arbeitsblätter, Filmdokumente, u.a.) sind dringend auf ihre sprachliche Barrierefreiheit kritisch zu überprüfen." (Wocken 2011, 127) Das heißt nicht, dass auf Materialien mit hohem sprachlichem Anspruch verzichtet werden muss. Es sollten aber Alternativen angeboten werden.

Ein wesentlicher Gelingensfaktor für die Umsetzung inklusiver Medienbildung ist die multidisziplinäre Kooperation. „An Inklusion orientierte Schulen sind auf den Austausch unterschiedlich ausgebildeter Lehrpersonen mit Eltern und weiteren Fachpersonen angewiesen." (Scholz 2012, 180) Dazu gehören unbe- dingt auch gut ausgebildete Medienpädagog/innen. Die Medienpädagogik kann einen wichtigen Beitrag für die Gestaltung inklusiver Unterrichtsarrangements leisten. „Gerade in diesem aktuellen Arbeitsfeld gilt es aber auch, Belastungssituationen und Bewältigungsmöglichkeiten ... angesichts der zu knappen personellen Ressourcen klar zu benennen." (Bosse u. a. 2009, 9) Wenn die Ausgestaltung inklusiver Medienbildung zum Beispiel durch an einer Schule vorhandene Ressourcen nicht möglich ist, kann eine sinnvolle Lösung in der Zusammenarbeit mit außerschulischen Kooperationspartnern liegen. Solche „Kooperationen [erschließen] allen Beteiligten Möglichkeiten zur wechselseitigen Annäherung und zur Erfahrung von mehr Selbstverständlichkeit im Umgang miteinander" (KMK 2012, 15). Bildungsinstitutionen haben vielfältige Möglichkeiten, Unterstützung durch Medienpädagog/innen von außen zu erhalten. Solche interdisziplinären Kooperationen werden oftmals auch finanziell unterstützt und bringen somit zusätzliche Ressourcen (zahlreiche Beispiele in Bosse 2013).

Der UN-Behindertenrechtskonvention geht es um die Umsetzung der Grund- und Menschenrechte von Menschen mit Behinderung, um die Verwirklichung gleichberechtigter Teilhabe in allen Lebensbereichen (UN 2006). Der aktuelle Medienkompetenzbericht macht deutlich, dass Medienkompetenz heute eine zentrale Voraussetzung zur Verwirklichung von Teilhabechancen darstellt (BMFSFJ 2013). Dazu bedarf es neben Medienpädagog/innen und Förderpädagog/innen mit spezifischen Kenntnissen vor allem einer breiten medienpädagogischen Grundbildung für pädagogische Fachkräfte in allen Institutionen entlang der Bildungskette. ,Medienkompetenzförderung gehört zunehmend in das Aufgabenprofil des pädagogischen Personals im Kontext der frühkindlichen Erziehung, der Schulen, aber auch der außerschulischen Jugendarbeit. (...) Nunmehr geht es darum si- 
cherzustellen, dass dieses medienpädagogische Anforderungsprofil vom gegenwärtigen und zukünftigen pädagogischen Personal auch erfüllt werden kann.“ (BMFSFJ 2013, 16) Im Zeitalter der Inklusion ist damit auch der gesellschaftliche und politische Anspruch verbunden, die Belange und Bedürfnisse beeinträchtigter, benachteiligter und behinderter Kinder und Jugendlicher zu berücksichtigen (Deutscher Bundestag 2012).

\section{Anmerkungen}

- Einen schnellen Überblick über zertifizierte Websites findet man auf der Homepage der Schweizer Stiftung „Zugang für alle" (www. access-for-all.ch). Die Stiftung bietet darüber hinaus einen Accessibility Quicktest an.

2 Eine Online-Datenbank zu unterstützenden Technologien findet sich unter www.barrierefrei-kommunizieren.de/datenbank.

\section{Literatur}

BMFSFJ / Bundesministerium für Familie, Senioren, Frauen und Jungend (2013): Medienkompetenzförderung für Kinder und Jugendliche. Eine Bestandsaufnahme. Online unter: www. bmfsfj.de/RedaktionBMFSFJ/Broschuerenstel le/Pdf-Anlagen/Medienkompetenzf_C3_B6rd erug-f_C3_BCr-Kinder-und-Jugendliche,prope rty $=p d f$, bereich $=b m f s f j$, sprache $=$ de, $r w b=$ true .pdf, 9.12.2013

Booth, T.; Ainscow, M. (2003): Index für Inklusion. Halle-Wittenberg: Martin-Luther-Universität. Online unter: www.eenet.org.uk/resources/ docs/Index\%20German.pdf, 30.11.2013

Bosse, I. (Hrsg.) (2013): Medienbildung im Zeitalter der Inklusion. LfM Dokumentation. Band 45. Düsseldorf: LfM

Bosse, I.; Hedderich, I.; Tscheke, J. (2009): Förderschwerpunkt körperlich-motorische Entwicklung? Bericht aus dem Workshop. In: VDS-Landesverband Sachsen e.V.: Landesmitteilung Heft 1, 8-14

Caspers, T. (2011): Einführung in Barrierefreiheit. Online unter: www.einfach-fuer-alle.delartikell einfuehrung-barrierefreiheit/, 6.6.2012
Deutscher Bundestag - Enquete Kommission Internet und Digitale Gesellschaft (2012): Projektgruppe Bildung und Forschung. Handlungsempfehlungen. Stand 25.6.2012. Online unter: http://www.bundestag.de/internetenquete/dokumentation/Sitzungen/201206 25/A-Drs_17_24_052___PG_Bildung_und_ Forschung_Handlungsempfehlungen.pdf, 31.7.2012

Florian, L.; Hegarty, J. (2004): ICT and special educational needs: A tool for inclusion. Berkshire: Open University Press

KMK/Kultusministerkonferenz (2012): Medienbildung in der Schule. Beschluss der Kultusministerkonferenz vom 8. März 2012. In: www. kmk.org/fileadmin/veroeffentlichungen_be schluesse/2012/2012_03_08_Medienbildung. pdf, 10.9. 2012

Körner, I. (2006): Stellenwert von Medienprojekten. In: Michaelis, E.; Lieb, O. (Hrsg.): Ausdrucksstark: Modelle zur aktiven Medienarbeit mit Heranwachsenden mit Behinderung. München: kopaed, 11

Malina, B. (Hrsg.) (2009): Leitlinien für die Bildungspolitik. Deutsche UNESCO Kommission e.V. Bonn: DUK

Maurer, B.; Reinhard-Hauck, P.; Schluchter, J.-R.; V. Zimmermann, M. (Hrsg.) (2013): Medienbildung in einer sich wandelnden Gesellschaft. München: kopaed

Michaelis, E.; Lieb, O. (2006): Ausdrucksstark: Modelle zur aktiven Medienarbeit mit Heranwachsenden mit Behinderung. München: kopaed

Ottinger, G. (2008): Soziale Ungleichheit in der Informationsgesellschaft. Das Phänomen der „digitalen Kluft" und die Bedeutung für die Sozialarbeit. Duisburg: WiKu-Verlag

Richiger-Näf, B. (2010): ICT und Sonderpädagogik. In: Schweizerische Zeitschrift für Heilpädagogik 16 (6), 6-11

Schnaak, T.; Böhmig, S. (2012): Inklusive Medienpädagogik - was ist das? In: Landesarbeitsgemeinschaft Lokale Medienarbeit NRW in Kooperation mit der Technischen Jugendfreizeit- und Bildungsgesellschaft (Hrsg.): Materialien für eine inklusive Medienpädagogik, 17-21

Scholz, D. (2012): Schüler mit körperlicher und motorischer Behinderung. In: Stangier, S.; Thoms, E.-M. (Hrsg.): Eine Schule für alle. Inklu- 
sion umsetzen in der Sekundarstufe. Mühlheim: Verlag an der Ruhr

Schorb, B.; Wagner, U. (2013): Medienkompetenz - Befähigung zur souveränen Lebensführung in einer mediatisierten Gesellschaft. In: Bundesministerium für Familie, Senioren, Frauen und Jugend/BMFSFJ: Medienkompetenzförderung für Kinder und Jugendliche. Eine Bestandsaufnahme. Online unter: www.bmfsfj.del RedaktionBMFSFJ/Broschuerenstelle/PdfAnlagen/Medienkompetenzf_C3_B6rderug$f_{-} C_{3}$ BCr-Kinder-und-Jugendliche, property= pdf, bereich $=$ bmfsfj, sprache $=d e, r w b=$ true.pdf, 9.12. 2013, 18-23

Stephanidis, C. (2001): User interfaces for all concepts, methods, and tools. Mahwah: Lawrence Erlbaum Associates

Tulodziecki, G. (2010): Medienbildung in der Schule. In: Bauer, P.; Hoffmann, H.; Mayrberger, K. (Hrsg.): Fokus Medienpädagogik. Aktuelle Forschungs- und Handlungsfelder. München: kopaed, 45-61
UN/United Nations (2006): Convention on the Rights of Persons with Disabilities and Optional Protocol. New York

UN/United Nations (2008): Übereinkommen über die Rechte von Menschen mit Behinderungen. Online unter: www.un.org/Depts/german/ueber einkommen/ar61 106-dbgbl.pdf, 9.12.2013

Wocken, H. (2011): Das Haus der inklusiven Schule. Baustellen - Baupläne - Bausteine. Hamburg: Feldhaus

\section{Anschrift des Autors}

\section{Jun. Prof. Dr. Ingo Bosse}

TU Dortmund

Fakultät Rehabilitationswissenschaften

Emil-Figge-Straße 50

D-44221 Dortmund

+49 (o) 2317554572

ingo.bosse@tu-dortmund.de 